\title{
Perpetual Environmentally Powered Sensor Networks
}

\author{
Xiaofan Jiang, Joseph Polastre, and David Culler \\ Computer Science Department \\ University of California, Berkeley \\ Berkeley, CA 94720 \\ xjiang@berkeley.edu, \{polastre,culler\}@cs.berkeley.edu
}

\begin{abstract}
Environmental energy is an attractive power source for low power wireless sensor networks. We present Prometheus, a system that intelligently manages energy transfer for perpetual operation without human intervention or servicing. Combining positive attributes of different energy storage elements and leveraging the intelligence of the microprocessor, we introduce an efficient multi-stage energy transfer system that reduces the common limitations of single energy storage systems to achieve near perpetual operation. We present our design choices, tradeoffs, circuit evaluations, performance analysis, and models. We discuss the relationships between system components and identify optimal hardware choices to meet an application's needs. Finally we present our implementation of a real system that uses solar energy to power Berkeley's Telos Mote. Our analysis predicts the system will operate for 43 years under $1 \%$ load, 4 years under $10 \%$ load, and 1 year under $100 \%$ load. Our implementation uses a two stage storage system consisting of supercapacitors (primary buffer) and a lithium rechargeable battery (secondary buffer). The mote has full knowledge of power levels and intelligently manages energy transfer to maximize lifetime.
\end{abstract}

\section{INTRODUCTION}

An essential element of the sensor network vision is the creation of sustainable computing - nodes that run perpetually using ambient energy in their physical environment. In outdoor settings, the most accessible environmental energy source is solar. While photo-voltaic (PV) power systems are in widespread use in many settings, the design of a PV system for perpetual operation of ultra-low power wireless sensor nodes presents a number of unique challenges. It should be simple, robust, and operate with no human intervention for many years. Duty cycle and power requirements are low for sensor networks, but the load varies over a huge range-microwatts in standby and milliwatts when active. Many applications operate at low duty cycles in unpredictable environments, so the system should adapt to the available energy reserve. Finally, the physical deterioration of the energy storage device is generally the overall limiting factor of lifetime of the device. For example, rechargeable batteries have about 300 to 500 recharge cycles, resulting in at most one to two years of operation if charged daily. This paper presents the design and implementation of Prometheus, an extremely long duration solar power subsystem for the most recent wireless sensor network mote-Telos [1]. The key design challenge is reducing the strain on storage elements while preserving a simple hardware and software architecture. We discuss the design of an intelligent system with lightweight and efficient hardware combined with powerful software that actively manages the power subsystem for perpetual operation.

\section{BACKGROUND AND RELATED WORK}

Our architecture, presented in Figure 1, reflects most environmental power systems in existence today (see [2] and [3] for more systems). They consist of four main components: an energy source, buffer, charge controller, and consumer. An energy source provides a certain amount of current under particular environmental conditions, such as solar energy. An energy consumer, such as a wireless sensor node

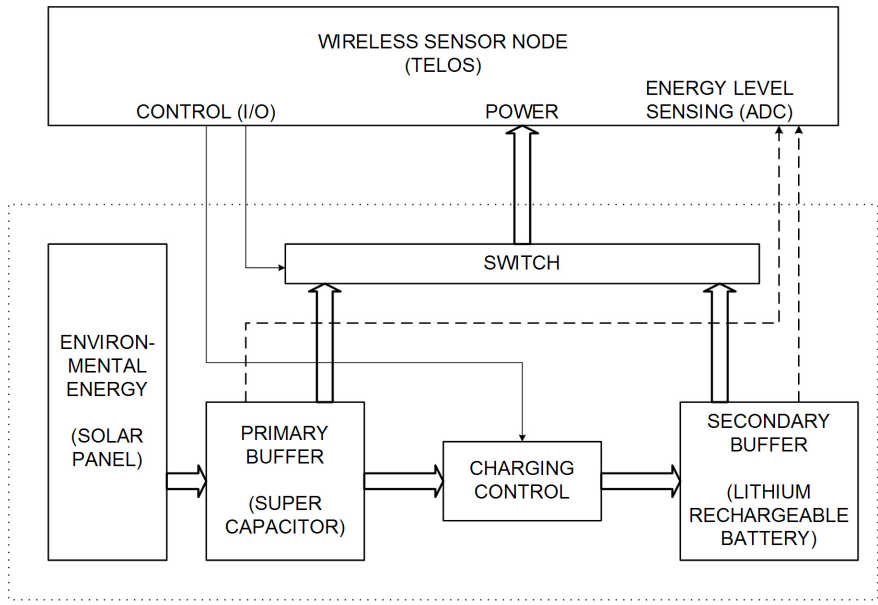

Fig. 1. System Architecture and Prometheus Implementation

like the Telos mote, has various operational modes where each mode may have an order of magnitude different current consumption. An energy buffer accumulates charge during periods of ample energy source and delivers charge during the remainder. Energy buffers are typically capacitors, supercapacitors, or rechargeable batteries. Finally, a charge controller replenishes buffers and provides the desired voltage or current to a consumer.

Several research efforts have prototyped the use of environmental energy to power wireless sensor networks [4], [5], [6], [7]. We drew on a design by UCLA described in [4]. It powered the earlier MICA mote [8], which has a more demanding power profile than the Telos mote used in our system. The UCLA design has only a secondary buffer consisting of a NiMH rechargeable battery and simple hardware to control energy transfers. Since solar energy directly enters the battery, it experiences recharge cycles daily placing significant stress on the battery. This limits the system's lifetime to no more than two years. Such a lifetime is not dramatically larger than that obtainable with batteries alone and far from perpetual operation. PicoRadio [5] considered rechargeable batteries but dismissed them due to limited recharge cycles. Instead, the system only used capacitors. When the energy source disappeared, the system experienced outages within only a few hours. MIT's Cricket [7] includes a capacitor to buffer current surges but does not operate without constant solar energy input. Our system addresses the recharge cycles concern through advanced charging control. Because the wireless node is carefully designed for low power operation, its load is far lower than and does not require the complex and energy consuming power control logic in ZebraNet [6]. The EE community has researched hybrid BattCap designs combining supercap and battery on the chemical level. However, for low cost high power systems, NiCad combined 
with large capacitors is the usual approach, which is inadequate for space-constrained sensor networks.

\section{Design AND ANALYSIS}

Figure 1 shows our implementation of a generic architecture that uses dual buffers and permits intelligent energy transfers. It addresses a range of environmental and application requirements by sizing the hardware components appropriately and through software support on the microcontroller (discussed in Section IV-E). The primary operating mode of our system is to use a volatile primary buffer to collect environmental energy and to power the sensor node, while using a second buffer as a reliable emergency backup.

\section{A. Environmental Energy Source}

Solar energy is one of the most abundant and accessible types of environmental energy. However, in most latitudes we only expect a few hours of direct sunlight, so a large buffer is needed to power the node through the night.

Solar cells come in various sizes providing different voltages and currents. We can wire them in parallel to increase current or in series to increase voltage. In general, increasing the area or light intensity produces a proportionate increase in current. Typical solar cell efficiency is around 18\% [3], corresponding to power output of about $18 \mathrm{~mW} / \mathrm{cm}^{2}$ under direct sunlight.

If we fix the voltage by choosing a configuration satisfying the voltage requirement of the system, we can model the environmental energy source simply as a power source:

$$
P_{E}(t)=P_{u n i t}(t) * A
$$

where $P_{\text {unit }}(t)$ is power per area or per solar panel, and $A$ is the area or number of solar panels connected in parallel.

For 6 hours of direct sunlight and 18 hours of darkness, the model becomes a pulse wave of $25 \%$ duty cycle with magnitude equal to the maximum power generated.

Since the energy capacity of the primary buffer is finite, a very high $P_{E}(t)$ is unnecessary. We only need enough energy to charge the primary buffer. The size of the solar panel should be determined based on how fast the primary buffer should be replenished; larger solar cells only yield quicker charging.

\section{B. Wireless Sensor Node}

The sensor node influences the system's power consumption by changing its duty cycle. It can be modeled as a power sink of periodic pulses. The power is dependent on three parameters: duty cycle $D$, active mode current $I_{\text {active }}$, and sleep mode current $I_{\text {sleep }}$. In most cases, we are only interested in the average power consumption (assuming wakeup time is negligible):

$$
P_{C_{A V G}}=V_{\text {supply }} *\left(D * I_{\text {active }}+(1-D) * I_{\text {sleep }}\right)
$$

Eq2 implies that $I_{\text {active }}, I_{\text {sleep }}$, and $V_{\text {supply }}$ should be as low as possible when selecting a wireless sensor node. $I_{\text {sleep }}$ is often negligible for sensor nodes. $D$ is chosen by the application, therefore if the application knows the energy levels of the two buffers, it can adjust duty cycle intelligently. Furthermore, when in a network, this information can be shared across nodes to make routing decisions (Section IV-E).

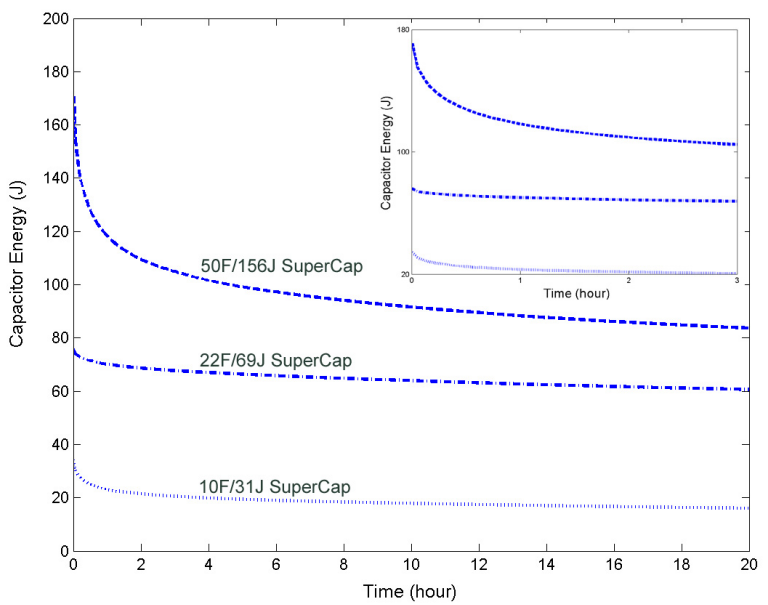

Fig. 2. Self-Discharge of Supercapacitors

\section{Primary Buffer}

The primary buffer needs to handle high levels of energy throughput and frequent charge cycles since it buffers volatile inputs from the energy source. The active load is large compared to the average load, so the primary buffer incurs a recharge cycle on every duty cycle of the node. Rechargeable batteries are typically rated for a few hundred charge cycles and, although they can endure many more shallow charge cycles than the advertised rating, their lifetime is significantly decreased by frequent charge cycles. Capacitors have virtually infinite recharge cycles and are ideal for frequent pulsing applications. Historically capacitors are rarely used as primary power due to their limited capacity, but large capacity super-capacitors are now a viable option. Unfortunately, super-capacitors have higher leakage current, larger size, and cost. The capacitor must provide energy to the consumer most of the time and minimize access to the secondary buffer to prolong its lifetime. Supercapacitors are the only option that meets this goal without deteriorating over time itself.

Supercapacitors vary from millifarads to hundreds of farads. To prolong the lifetime of the secondary buffer, the primary supercap should be as large as possible. Unfortunately, the larger the capacity, the greater the leakage current; this is continuously flowing current that returns to ground through a capacitor. To determine the optimal capacitance, depending on the leakage and the consumption level, we first model the primary buffer as an energy source:

$$
E_{1}(t)=\max \left(\int_{t}\left(P_{I N}(t)-P_{O U T}(t)-P_{L E A K}(t)\right) d t, E_{\text {max }}\right)
$$

where $P_{I N}$ is the power from the environmental energy source. $P_{I N}$ may only be a fraction of $P_{E}$ because the voltage of $P_{I N}$ is capped by the maximum input voltage of $E_{1}$. In other words, $P_{I N}=\frac{V_{E 1 \max }}{V_{P E}} P_{E} . P_{O U T}$ is the output, and $P_{L E A K}$ is the internal leakage.

The precise leakage function $P_{L E A K}$ often needs to be determined experimentally, as they are only crudely specified in the data sheets. Figure 2 shows the leakage pattern of three supercapacitors we tested under isolation. They all experience rapid leakage when fully charged.

To find the theoretical optimal capacitance, let us set $P_{I N}=0$ and use the leakage data in Figure 2 The initial energy in the supercapacitors $\left(\frac{1}{2} C V_{\max }^{2}\right)$ is the initial condition of $\int_{t} P_{I N}(t)$. 


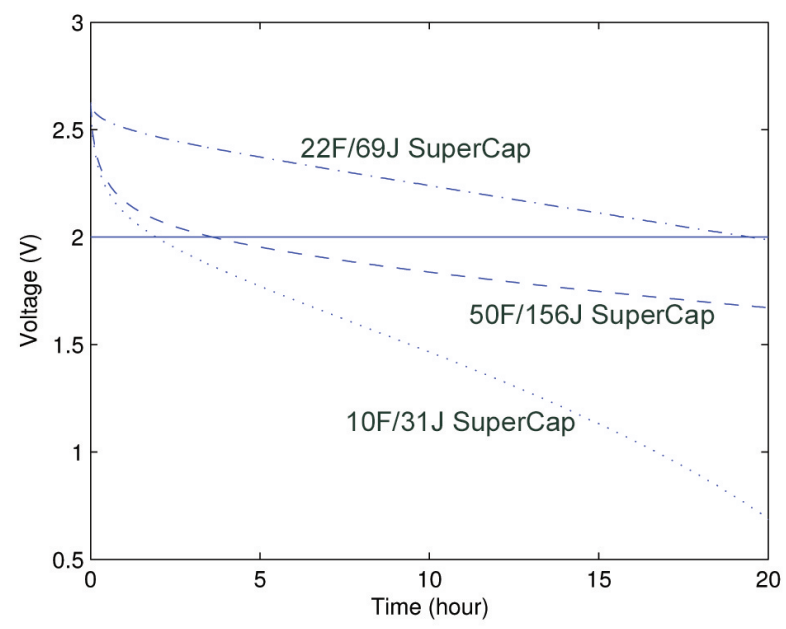

Fig. 3. Supercapacitor leakage under load

\begin{tabular}{l|l|l|l|l|l|l} 
Type & $\begin{array}{l}\text { Op. } \\
\text { Volt. }\end{array}$ & Memory & $\begin{array}{l}\text { Density } \\
(\mathrm{Wh} / \mathrm{kg})\end{array}$ & Cycle & $\begin{array}{l}\text { Leakage } \\
(\% / \text { Month })\end{array}$ & Charging \\
\hline NiCad & 1.2 & Yes & 50 & $1200^{*}$ & 15 & Simple \\
NiMH & 1.2 & Yes & 70 & 300 & 30 & Simple \\
Lithium & 3.7 & No & $\begin{array}{l}100+\quad 500 \\
\text { Deep cycles }\end{array}$ & $\begin{array}{l}\text { Complex } \\
\text { De }\end{array}$
\end{tabular}

Fig. 4. Rechargeable Battery Comparison

Figure 2 can be represented by the equation

$$
\int_{t}\left(P_{I N}(t)-P_{L E A K}(t)\right) d t=\frac{1}{2} C V_{\max }^{2}-\int_{t} P_{L E A K}(t) d t
$$

Let us assume the consumer has the power consumption pattern of Eq2 where $V_{\text {supply }}(t)$ is the voltage of $E_{1}(t), P_{O U T}$ becomes $P_{C_{A V G}}=\sqrt{2 \frac{E_{1}(t)}{C}} I_{A V G} . \mathrm{Eq} 3$ becomes

$$
E_{1}(t)=\left[\frac{1}{2} C V_{\max }^{2}-\int_{t} P_{L E A K}(t) d t\right]-\int_{t} \sqrt{2 \frac{E_{1}(t)}{C}} I_{A V G} d t
$$

Since the node dies when supply voltage goes below a minimum value, we are interested in the graph of $V_{1}(t)=\sqrt{2 \frac{E_{1}(t)}{C}}$. For a consumer with a duty cycle of $1 \%$, active current of $10 \mathrm{~mA}$ and negligible sleep current, $V_{1}(t)$ is graphed in Figure 3

Choosing the best capacitance means maximizing $t$ while keeping $V(t)$ above the minimum operating voltage. From Figure 3 we observe that bigger capacitance is not better-22F performs better than both $10 \mathrm{~F}$ and $50 \mathrm{~F}$.

Configuration of supercapacitors also play an important role in maximizing $t$ of $V_{1}(t)$. Configuration refers to series or parallel combination of supercapacitors. The leakage of supercapacitors is proportional to the energy level (or quadratically proportional to voltage since $E=\frac{1}{2} C V^{2}$ ). We can lower leakage by wiring two supercapacitors in series. This results in half the total capacitance, but the decrease in leakage is greater due to the quadratic dependence on voltage. Wiring the capacitors in parallel increases capacitance, but the increase in leakage makes this solution impractical. More complex configurations such as parallel of series of capacitors or vise-versa are also possible but are not desirable due to greater leakage.

\section{Secondary Buffer}

When the primary buffer is exhausted, the secondary buffer is used. It needs to hold energy for a long period of time and have

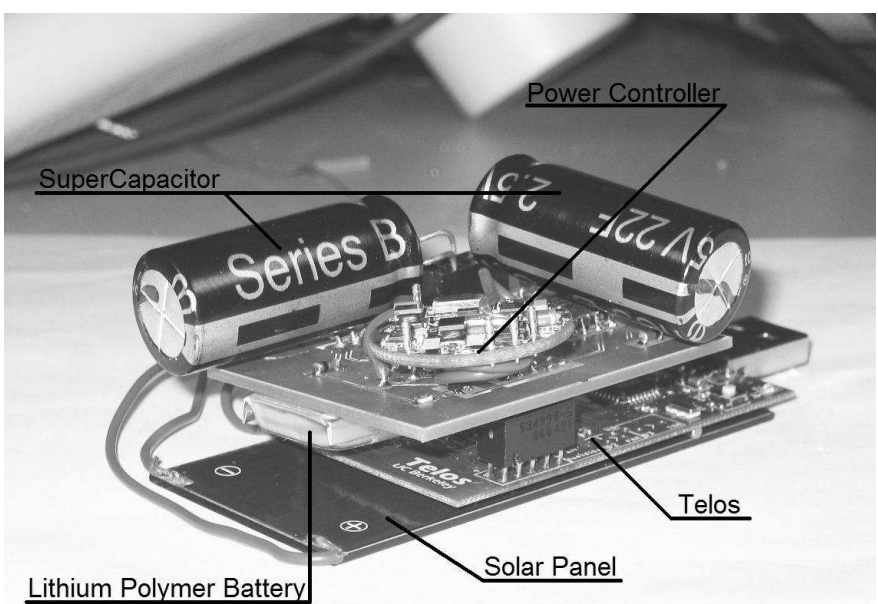

Fig. 5. Prometheus: Perpetual Self Sustaining Telos Mote

low leakage but not charged or discharged frequently. Rechargeable batteries meet many of these requirements. There are several types including NiCad, NiMH, and Lithium (Ion / Polymer), each with advantages and disadvantages shown in Figure 4

Lithium rechargeable has the lowest leakage, highest density, high recharge cycles, and provides high voltage with a single cell. However, more complex charging circuits are required to prevent harmful effects that reduce the lifetime of the battery.

While the primary buffer is charged by environmental energy, the secondary buffer can be charged either by environmental energy or by the primary buffer. If the secondary buffer is NiMH or NiCad, it is possible to charge it directly using environmental energy to reduce complexity. For lithium batteries, they must be charged from the primary buffer where energy is stable and pulsing is possible. Charging could be done either using entirely hardware, such as dedicated charging chip, or using a combination of software and hardware. Charging chips are designed for laptops and use Coulomb counters which require the chip to be always on. This power consumption is greater than the battery leakage and is not tolerable. Instead, software enables more complex schemes that prolong the secondary buffer's lifetime.

\section{IMPLEMENTATION}

We implemented our energy transfer system on a prototyping board that connects to the Berkeley Telos sensor through its 10-pin connector. As seen in Figure 5 our board replaces the battery pack with a solar panel, two supercapacitors, and a small Li+ battery. The block diagram of our system is shown in Figure 1 The component choices and their characteristics are described in this section.

\section{A. Hardware Selection}

We used Sunceram's $37 \times 82 \mathrm{~mm}$ solar panel [9] due to its large availability and low price ( $\$ 15$ retail). The $37 \times 82 \mathrm{~mm}$ panel fits our Telos nicely and its $4.5 \mathrm{~V}$ output matches the $5 \mathrm{~V}$ maximum voltage of our primary buffer.

We used supercapacitors from Aerogel [10] due to their relatively small leakage. They have a maximum voltage rating of $2.5 \mathrm{~V}$, but our solar panel outputs $4.8 \mathrm{~V}$. Instead of using a diode to cap the voltage, we wired two supercapacitors in series to reduce leakage. If we operate Telos at $1 \%$ duty cycle, the average power consumption is $\frac{20 m A+99 * 5 u A}{100}=205 u A$. Using Figure 3, we chose 22F supercapacitors. 


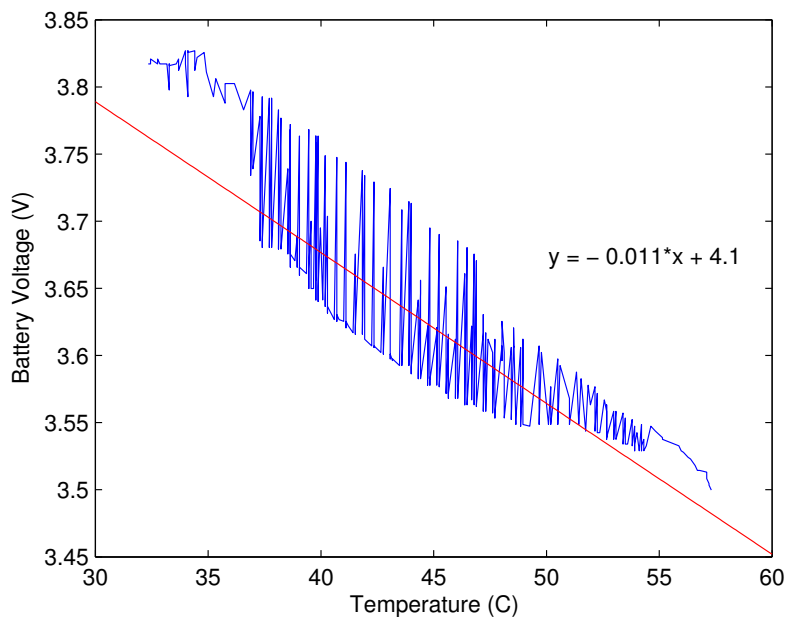

Fig. 6. Battery Voltage vs. Temperature: Battery readings must be compensated with temperature information in order for the node to know the true battery capacity

Batteries are often the limiting factor of a node's lifetime. Therefore we chose lithium because it has a large number of recharge cycles, high charge density, low leakage, lack of memory effect, and provides sufficient voltage with one cell (see Figure 47. We found that shallow discharge/charge cycles can extend the battery's life. Software optimizes charging to utilize this behavior as discussed in Section IV-E

The voltage of the battery will decrease linearly with increasing temperature (see Figure 6). This may trigger a charge when not necessary. Software can compensate for the drop in voltage using the built-in temperature sensor on our node (see Section IV-E).

Due to the high density of lithium batteries, we chose a small ( 0.5 in $x$ lin) battery. A capacity of around $500 \mathrm{mAh}$ is suitable for our system; however, we used a $200 \mathrm{mAh}$ lithium polymer battery to obtain quicker results. Larger capacities extend the total lifetime.

\section{B. Telos Wireless Sensor Node}

We chose Berkeley's Telos Mote because it can operate at extremely low voltages $(1.8 \mathrm{~V})$, extracting as much energy as possible from the supercapacitors. It also has the lowest $I_{\text {active }}, I_{\text {sleep }}$, and wakeup time of any wireless sensor node (see Section III-B and [1]). Telos draws $20 \mathrm{~mA}$ in active mode and $5 \mathrm{uA}$ in sleep mode. We use two ADC channels and two I/Os to monitor and control the power board. In Telos Revision B, one set of ADC and I/O will be replaced by Telos' internal supply power supervisor.

\section{Sensing and Control}

The Telos ADC monitors the energy levels (voltages) of the supercapacitor and the battery. Instead of continuously monitoring the voltage levels in hardware (which consumes energy), we "piggyback" a reading on every duty cycle of the application. Applications with an active period at least once an hour is sufficient. This is a simple yet cost-effective approach. However, because the internal reference voltages of Telos are only $2.5 \mathrm{~V}$ and $1.5 \mathrm{~V}$, we need two voltage dividers to drop the maximum $4.8 \mathrm{~V}$ down to less than $2.5 \mathrm{~V}$. A set of larger resistance resistors (such as $1 M \Omega$ ) would be less accurate than smaller resistance (such as $1 k \Omega$ ) but consumes less power. We chose $1 M \Omega$ resistors with $1 \%$ error because a few millivolts of error is tolerable.
The energy level information is used by Telos to directly control a digital switch to arbitrate the two buffers. Because the MAX4544 digital switch uses active elements consuming less energy than passive elements such as transistors, it was chosen for our system. It interfaces with the digital I/O pins on Telos.

\section{Charging Circuitry}

We use a MOS switch with a simple DC/DC converter (used to limit current) to minimize power lost to charging hardware, as shown in Figure 7 on the next page. This is possible because software has complete control over the charging process. When the battery is below a certain level and conditions are met as indicated by software, we replenish the battery with energy from the supercapacitor. Charging lithium batteries requires a constant pulsing current until charged to $80 \%$ of its full capacity. Software can control the battery level to stay in this charging region. Using a dedicated battery charging chip is unnecessary as it includes functionalities not needed and raises costs and power consumption. Our DC/DC converter can perform the same function since the MAX1676 has an internal current limiter (selectable at $.5 \mathrm{~A}$ and $1 \mathrm{~A}$ ). A charging current of around $1 \mathrm{x}$ the capacity of the battery $(500 \mathrm{mAh} \rightarrow .5 \mathrm{~A}$ charging current) is considered safe. We used a P-channel MOSFET as the switch instead of a digital switch because small digital switches cannot handle large currents.

\section{E. Driver and Software}

In order to simplify the hardware design, we pushed control logic to the Telos MCU. By doing so, we reduced the number of physical components and quiescent current consumption. Software has complete control over buffer selection and charging. A driver for our energy transfer system is shown below that uses simple if-else statements yet utilizes the power of the microprocessor to intelligently manage the switching and charging process to prolong the lifetime of the node (corresponding code shown in parenthesis):

1. Compensate for drop in battery voltage due to rise in temperature (1-2).

2. Provides hysteresis between the supercapacitor and battery to avoid unnecessary access to battery (3-6).

3. Charge only when excess energy (direct sunlight) is detected in primary buffer (7-10).

4. Stop charging as soon as direct sunlight is gone even though there is plenty of energy left in supercapacitors. This allows Telos to survive for the rest of the day and night without resorting to battery. The supercapacitors will be charged again the next day (7-10).

5. Report status and energy levels to protocols / services (11).

\section{PROMETHEUS DRIVER}

1. if $T e m p V>2.2$

2. $B a t t V=B a t t V+1.45 *($ TempV -2.2$)$

3. if $C a p V<2.2$

4. SwitchCap $=$ FALSE

5. if $C a p V>3.5$

6. SwitchCap = TRUE

7. if $C a p V>4.4$ and $B a t t V<3.5$

8. $\quad$ ChargeBatt $=$ TRUE

9. if $C a p V<3.8$

10. ChargeBatt $=$ FALSE

11. call Radio.send(STATS) 


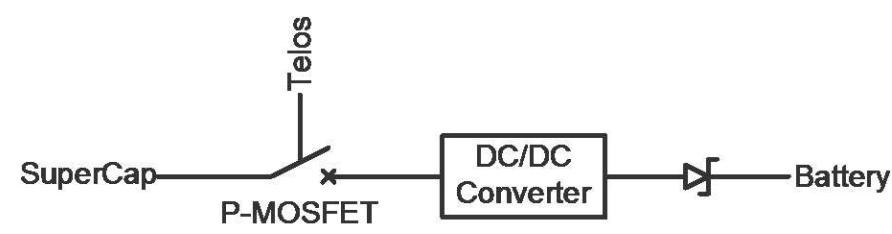

Fig. 7. Block Diagram of Charging Circuit

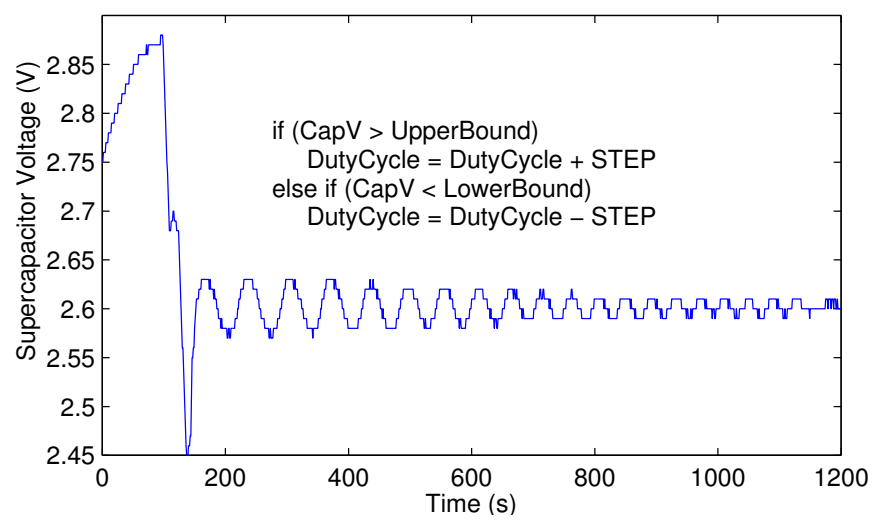

Fig. 8. Duty-Cycle Adaptation

Sophisticated schemes may be implemented to adapt to different operating conditions. Higher level software can take advantage of energy knowledge. One example is to dynamically adjust duty cycle based on the energy in the primary buffer. When there is sufficient energy, Telos runs at a higher duty cycle, and when the energy is low, it runs at a lower rate. Figure 8 shows Telos adjusting its duty cycle until it is fully utilizing the environmental energy. This is useful when the environmental energy is distributed unevenly in the network (such as spots of sun light through trees). Nodes with higher exposure to environmental energy will increase their duty cycle to do more work (such as routing packets) while less exposed nodes will only perform minimal tasks.

\section{Results AND ANALYSis}

Our $37 \mathrm{x} 82 \mathrm{~mm}$ solar panel generates $40 \mathrm{~mA}$ at $4.8 \mathrm{~V}$ under direct sunlight and fully charges the supercapacitors in less than two hours as shown in Figure 10 A from $\mathrm{t}=4$ to $\mathrm{t}=6$.

We tested our system using the driver in Section IV-E running at $1 \%$ duty cycle. The first scenario is during the night and the energy in the supercapacitors is low. Our system should switch to secondary buffer to sustain operation. The second scenario is during sunrise. Our system should initiate a charge to the battery when supercapacitor has excess energy since the system started with a low battery level.

In the first scenario (Figure 9), our system started at night when the supercapacitor is at $3 \mathrm{~V}$ (two combined), battery at $3.4 \mathrm{~V}$, and reference voltage at $2.5 \mathrm{~V}$. After 1.6 hours $(\mathrm{t}=1.6)$, the capacitor voltage drops below 2.6 and Telos changes its reference voltage to $1.5 \mathrm{~V}$ since the $2.5 \mathrm{~V}$ reference is no longer sustainable. This causes the reported battery voltage to clip to $3 \mathrm{~V}$. After another 4.2 hours $(\mathrm{t}=5.8)$, the capacitor drops below $2.2 \mathrm{~V}$ and triggers Telos to switch from primary to secondary buffer. Since the battery is at $3.4 \mathrm{~V}$, the reference is set back to $2.5 \mathrm{~V}$.

In the second scenario (Figure 10, the system initially runs on battery (as seen in 10 C). After 4 hours, the sun comes out and starts to charge the supercapacitors (as seen in 10 A starting at $t=4$ ). At
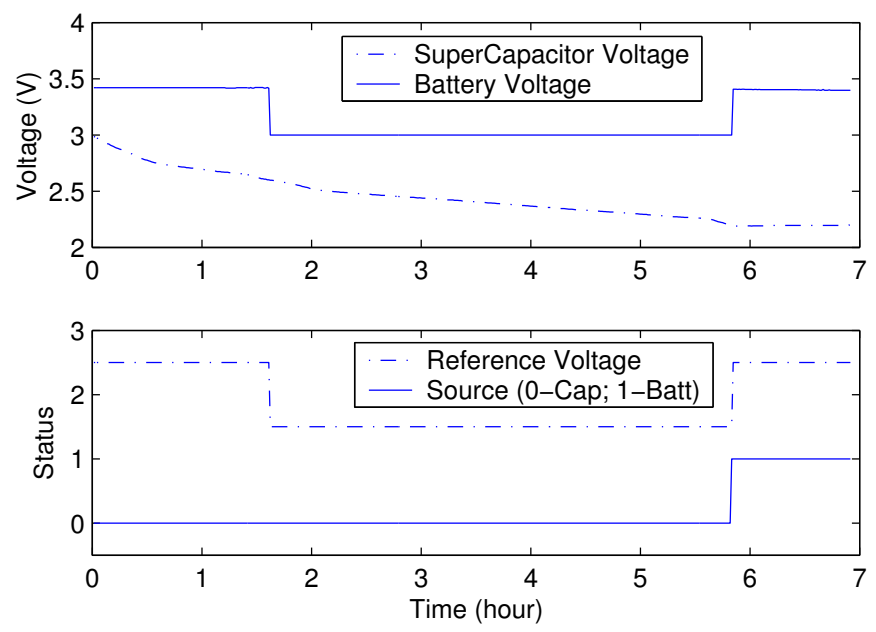

Fig. 9. Telos switches from Primary Buffer to Secondary Buffer and adjusts its reference voltage

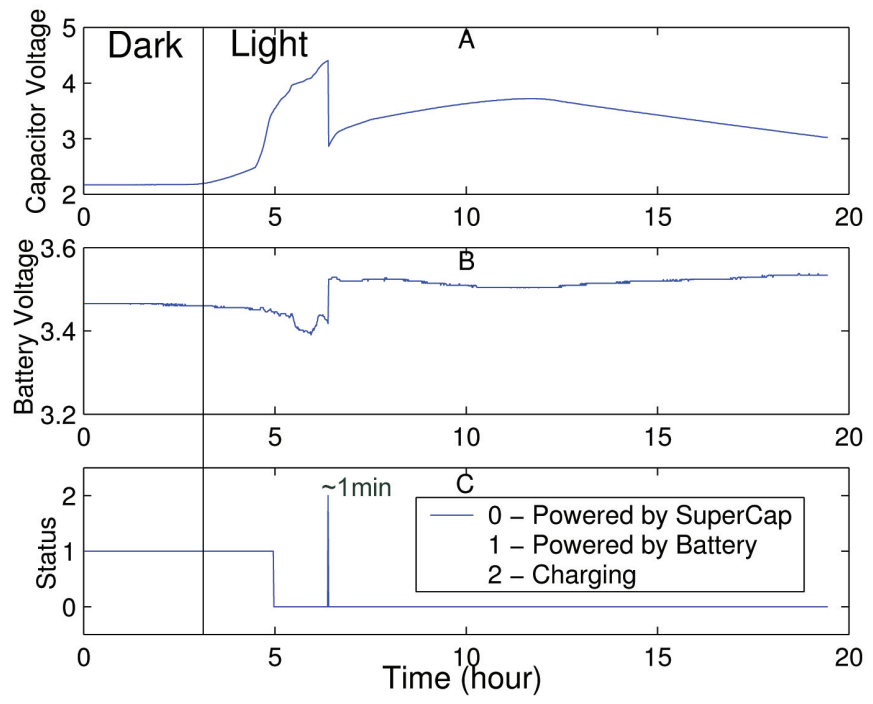

Fig. 10. Telos charges lithium battery from supercapacitors

4.8 hours, the capacitor voltage exceeds $3.5 \mathrm{~V}$, switching power back from battery to supercapacitors. At around 6.5 hours, the capacitor voltage exceeds the charging threshold of $4.4 \mathrm{~V}$ and the battery is below $3.5 \mathrm{~V}$, resulting in a charge pulse (as seen by the sharp drop in capacitor voltage in A and increase in battery voltage in B). This rapidly transfers most of the supercapacitors' energy to the battery. The solar panel quickly replenishes the supercapacitors and battery voltage stabilizes at around $3.54 \mathrm{~V}$.

We let the system run for another 10 days and Telos has not yet resorted to battery (except the first day shown in Figure 10 on which we intentionally initialized the supercapacitors at a low energy level).

Under continuous low light conditions (assuming no light), the estimated time to outage for our system (200mAh battery) is $\frac{0.2 \mathrm{Ah}}{\left(205 \times 10^{-6} \mathrm{~A}\right) \times 24 \text { hours }}=40.65$ days. A larger battery results in proportionally longer time. This calculation implies that as long as the light source does not stay extremely low for months, our system should be able to continuously operate. The operation is not just limited by the energy generation and consumption, but also the 


\begin{tabular}{|l|l|l|}
\hline Duty Cycle & Required Light & Life Time \\
\hline $1 \%$ & $5 \mathrm{hrs} /$ month & $43 \mathrm{yrs}$ \\
$10 \%$ & $5 \mathrm{hrs} / 4 \mathrm{days}$ & $4 \mathrm{yrs}$ \\
$100 \%$ & $10 \mathrm{hrs} / 1$ day & $1 \mathrm{yr}$ \\
\hline
\end{tabular}

Fig. 11. Perpetual Operation: Predicted lifetime of the system components implemented with Prometheus, the effect of node duty cycle, and the required light to sustain operation.

stress placed on the system components. Figure 11 shows the trade off of duty cycle and lifetime and our predictions for how long the supercapacitors and lithium battery will operate before failing. By intelligent managing energy transfers, Prometheus may operate without human intervention or servicing. For most wireless sensor networks applications where duty cycle is $1 \%$ or less, our system provides perpetual operation.

\section{CONCLUSION}

We have presented an architecture for perpetual operation of wireless sensor networks using environmental energy. Our system intelligently manages a two-stage buffer to prolong the lifetime of the system hardware, including super-capacitor and lithium rechargeable battery. The energy level data collected by our sensor node may be used to build power-aware wireless networking protocols. We have demonstrated that our system works as predicted by our analysis and yields long-lived sensor network deployments.

\section{REFERENCES}

[1] J. Polastre, R. Szewczyk, and D. Culler, "Telos: Enabling ultra-low power research," in IPSN/SPOTS, Apr. 2005.

[2] J. Schaeffer and D. M. Pratt, The Real Goods Solar Living Sourcebook: The Complete Guide to Renewable Energy Technologies and Sustainable Living, 11st ed. Gaiam Real Goods, Aug. 2001.

[3] S. Roundy, D. Steingart, L. Frechette, P. K. Wright, and J. M. Rabaey, "Power sources for wireless sensor networks," in Proceedings of EWSN 2004, Berlin, GERMANY, Jan. 2004.

[4] A. Kansal, D. Potter, and M. B. Srivastava, "Performance aware tasking for environmentally powered sensor networks," in Sigmetrics 2004, 2004.

[5] S. Roundy, B. P. Otis, Y.-H. Chee, J. M. Rabaey, and P. Wright, "A $1.9 \mathrm{ghz}$ rf transmit beacon using environmentally scavenged energy," in IEEE Int.Symposium on Low Power Elec. and Devices, Feb. 2003.

[6] P. Zhang, C. M. Sadler, S. A. Lyon, , and M. Martonosi, "Hardware design experiences in zebranet," in SenSys 2004, Nov. 2004.

[7] H. Balakrishnan, R. Baliga, D. Curtis, M. Goraczko, A. Miu, B. Priyantha, A. Smith, K. Steele, S. Teller, and K. Wang, "Lessons from developing and deploying the cricket indoor location system," in MIT Technical Report, Nov. 2003.

[8] University of California, Berkeley, "Mica Platform," http://www.tinyos. net/scoop/special/hardware/ Sept. 2002.

[9] Panasonic, "Panasonic solar cells techinical handbook '98/99," http:// downloads.solarbotics.com/PDF/sunceramcat.pdf Aug. 1998.

[10] Cooper Industries, "Aerogel supercapacitors - b series data sheet," http: //www.cooperet.com/pdfs_html/PowerStorB_Specs.pdf Sept. 2002. 